\title{
Estudio de la propagación sexual del arboloco Montanoa quadrangularis Schultz Bipontianus Asteraceae
}

\author{
Marcela Tamayo-Rincón ${ }^{1}$, Loyla Rodríguez-Perez ${ }^{1 *}$, William Escobar-Torres ${ }^{2}$ \\ ${ }^{1}$ Centro de Investigaciones y Estudios en Biodiversidad y Recursos Genéticos (CIEBREG). Facultad de Ciencias. \\ Pontificia Universidad Javeriana. Bogotá, D.C. Colombia \\ ${ }^{2}$ Corporación Colombiana de Investigación Agropecuaria (CORPOICA). Santa Marta, Colombia \\ *loyla.rodriguez@javeriana.edu.co
}

Recibido: 19-02-2010: Aceptado: 04-05-2010

\begin{abstract}
Resumen
Objetivo. Desarrollar metodologías para la propagación sexual Montanoa quadrangularis Schultz Bipontianus (“Arboloco”). Materiales y métodos. Las semillas se colectaron de árboles establecidos en La Estación Ambiental de Chilaca (Geoambiente Ltda) localizada en el municipio de Pacho-Cundinamarca (Colombia). Se establecieron dos etapas de estudio: en semillero, se evalúo el efecto de los sustratos suelo local, mezcla suelo local con humus de lombriz y turba y dos arreglos espaciales: siembra directa al azar y a $0,5 \mathrm{~cm}$ de profundidad sobre la respuesta germinativa de las semillas. En vivero, se evaluó el efecto de los sustratos: suelo local y suelo local mezclado con humus de lombriz y turba, los estados de desarrollo de las plantas: dos hojas, cuatro hojas y seis hojas sobre el crecimiento y desarrollo vegetal. Resultados. En semillero, la turba determinó la mayor capacidad germinativa con 83,67\%, mientras que en el suelo local, se obtuvo la menor capacidad germinativa con un 40,83\%. En vivero, las plantas con 6 hojas y trasplantadas en el sustrato suelo local con humus de lombriz, presentaron las mejores respuestas en parámetros como longitud total por planta $(9,51 \mathrm{~cm})$, área foliar $\left(36,69 \mathrm{~cm}^{2}\right)$, el peso seco total de la planta $(0,10 \mathrm{~g})$. Conclusiones. El tipo de sustrato utilizado en semillero, afectó la capacidad germinativa de semillas de $M$. quadrangularis. El tipo de sustrato utilizado en vivero y el estado de desarrollo, incidieron significativamente en el crecimiento y desarrollo del arboloco.
\end{abstract}

Palabras clave: índices de germinación, crecimiento y desarrollo, especies forestales.

\begin{abstract}
Sexual propagation of arboloco Montanoa quadrangularis Schultz Bipontianus (Asteraceae). Objective. To develop sexual propagation methodologies for Montanoa quadrangularis Schultz Bipontianus (arboloco). Materials and methods. Seeds were harvested from trees grown at the Chilaca environmental station (Geoambiente Ltda.) located in Pacho, Cundinamarca, Colombia. The study was carried out in two phases: firstly, substrate effect on germination responses was evaluated in seedbed conditions. Substrates evaluated were: local soil, local soil mixed with worm humus, and peat. Additionally, two spatial arrangements were assessed: seeds sown at random and at $0.5 \mathrm{~cm}$ in depth. Secondly, in nursery conditions the effect of the substrates local soil, local soil mixed with worm humus and peat, as well as the effect of developmental stages of the plants (with 2, 4, and 6 leaves) on their further growth and development were evaluated. Results. In seedbed conditions, the germination capacity was highly influenced by peat substrate $(83.67 \%)$ whereas in local soil the germination capacity was $40.83 \%$. In nursery conditions, 6-leaf plants grown in local soil mixed with worm humus showed the best responses on parameters such as plant total length $(9.51 \mathrm{~cm})$, leaf area $\left(36.69 \mathrm{~cm}^{2}\right)$, and total dry weight $(0.10 \mathrm{~g})$. Conclusions. The type of substrate used in seedbed conditions influenced the germination capacity of $M$. quadrangularis seeds. The substrate and the developmental stage of the plant had a significant influence on the growth and development of arboloco plants.
\end{abstract}

Key words: germination indices, plant growth and development, forestry species. 


\section{Resumo}

Estudo da propagação sexual do "arboloco” Montanoa quadrangularis Schultz Bipontianus Asteraceae. Objetivo. Desenvolver metodologias para a propagação sexual da especie Montanoa quadrangularis Schultz Bipontianus (“Arboloco”). Materiais e métodos. As sementes foram coletadas de árvores localizadas na "La Estación Ambiental de Chilaca (Geoambiente Ltda), município de Pacho, Cundinamarca (Colômbia). Houve duas etapas de estudo: em sementeira, onde foi avaliado o efeito de substratos do solo local, mistura de solo local com húmus de minhoca e turfa e dois arranjos espaciais: plantio direito aleatório e a $0,5 \mathrm{~cm}$ de profundidade, sobre a resposta germinativa das sementes. No viveiro, foi avaliado o efeito dos substratos: solo local e solo local misturado com húmus de minhoca e turfa, e os estágios de desenvolvimento das plantas: duas folhas, quatro folhas e seis folhas no crescimento e desenvolvimento das plantas. Resultados. Na sementeira, a turfa determinou a maior capacidade germinativa com $83,67 \%$, enquanto que o solo local apresentou a menor capacidade germinativa com 40,83\%. No viveiro, as plantas com 6 folhas e transplantadas para o substrato de solo local com húmus de minhoca, apresentaram as melhores respostas em parâmetros como o comprimento total por planta $(9,51 \mathrm{~cm})$, área folhar $\left(36,69 \mathrm{~cm}{ }^{2}\right), \mathrm{e}$ peso da matéria seca total da planta $(0,10 \mathrm{~g})$. Conclusões. $\mathrm{O}$ tipo de substrato utilizado na sementeira afetou a capacidade germinativa das sementes de $M$. quadrangularis. O tipo de substrato utilizado no viveiro e o estado de desenvolvimento, influenciaram significativamente no crescimento e desenvolvimento do "Arboloco".

Palavras-chave: taxas de germinação, crescimento e desenvolvimento, espécies florestais.

\section{Introducción}

La biodiversidad constituye un patrimonio importante para la humanidad, teniendo en cuenta su beneficio directo y las funciones de soporte de las condiciones de vida sobre el planeta que cumplen los ecosistemas. Así mismo, el potencial genético y bioquímico que representan las especies vegetales puede ser aprovechado en beneficio de la humanidad. El Centro de Investigaciones y Estudios en Biodiversidad y Recursos Genéticos (CIEBREG) se interesa por estudiar el estado en el que se encuentran los bosques en el Complejo Ecoregional de los Andes del Norte, a partir del reconocimiento y valoración del potencial de los bienes y servicios ecológicos, para definir estrategias metodológicas para la propagación de especies forestales de interés económico, entre las que se encuentra el arboloco Montanoa quadrangularis Schultz Bipontianus. Con el propósito de mejorar la producción y calidad de las plantas de esta especie y aportar a los agricultores y entidades de fomento forestal herramientas de trabajo para la conservación y manejo sostenible del arboloco, se planteó este estudio, de la propagación por vía sexual en dos etapas: semillero y vivero, el cual evaluó la respuesta germinativa de las semillas y el crecimiento y desarrollo de las plantas de arboloco bajo diferentes condiciones ambientales.

M. quadrangularis es una especie forestal pionera de los ecosistemas intervenidos de los Andes tropicales de Colombia y Venezuela. El área de distribución de esta especie abarca las tres cordilleras andinas de Colombia, en altitudes comprendidas entre los 1300 y los 2800 msnm y con precipitaciones anuales que oscilan entre los $1300 \mathrm{y}$ $2500 \mathrm{~mm}(1,2)$.

Las plantas de arboloco se caracterizan por presentar un rápido crecimiento y una alta tasa de producción de biomasa, lo que determina su utilización en la recupera- ción de zonas degradadas por derrumbes, deforestación y erosión causada por las diferentes actividades humanas. En las etapas tempranas de la sucesión vegetal, se destaca la presencia de plantas de $M$. quadrangularis, especie de gran importancia etnobotánica, ya que produce una madera de buena calidad, resistente y de alta durabilidad, que es utilizada en la construcción de casas y en la elaboración de artesanías, así mismo se le reconocen usos medicinales $(2,3)$.

\section{Materiales y métodos}

\section{Población de estudio y muestra}

La población de estudio se obtuvo a partir de los frutos colectados de plantas de arboloco establecidas en la Estación Ambiental de Chilaca (Geoambiente Ltda). El establecimiento de las plantas de arboloco se realizó bajo condiciones ambientales de vivero en esta misma localidad. La Estación Ambiental Chilaca, se localiza en el municipio de Pacho (Departamento de Cundinamarca), a una altitud aproximada de $2050 \mathrm{msnm}$, con una temperatura promedio anual de $17^{\circ} \mathrm{C}$, una precipitación promedio anual de $1500 \mathrm{~mm}$ y una humedad relativa promedio de $82 \%$.

\section{Diseño experimental y Métodos}

Para el estudio de la propagación sexual de Montanoa quadrangularis Schultz Bipontianus se propusieron dos etapas: semillero y vivero. En la etapa de semillero, se evaluó el efecto del sustrato y del arreglo espacial sobre la respuesta germinativa de las semillas de $M$. quadrangulari. En la etapa de vivero, se evaluó el efecto del tipo de sustrato 
y el estado de desarrollo de las plantas al momento de trasplante sobre el crecimiento y desarrollo de las plantas de arboloco.

En la etapa de semillero, se aplicó un diseño de parcelas divididas con distribución completamente al azar, en el cual el factor principal fue el tipo de sustrato con tres niveles: suelo local (S1), mezcla de suelo local con humus de lombriz 1:1 v/v (S2) y turba (S3); el factor secundario fue el arreglo espacial con dos niveles: P1, siembra directa al azar y P2, semillas sembradas a distancias de $10 \mathrm{~cm}$ entre surcos, $5 \mathrm{~cm}$ entre semillas y $0,5 \mathrm{~cm}$ de profundidad. Los resultados del análisis de sustratos se presentan en la Tabla 1.

Las semillas se almacenaron durante 15 días bajo condiciones de oscuridad, siguiendo el protocolo propuesto por Torres (2004). Al final del período de almacenamiento, las semillas fueron sembradas en tres semilleros, los tratamientos tuvieron tres repeticiones de 100 semillas, para un total de 300 semillas por tratamiento.

Para evaluar la respuesta germinativa, el criterio de germinación se definió como la emergencia del gancho del hipocótilo (5). El análisis de la respuesta germinativa se realizó con base en los índices de germinación referidos por Thompson \& El-Kassaby (1993), los cuales se calcularon a partir del número de semillas germinadas obtenidas en los 30 días de muestreo:

Capacidad de germinación (GC): Este índice expresa el porcentaje de semillas germinadas al final de la prue- ba con respecto al número total de semillas puestas a germinar (7).

Índice de la tasa de germinación (GRI): Expresa la velocidad de germinación de acuerdo con el número total de semillas que germinan en un intervalo de tiempo (7). Se halla a partir de la fórmula:

$$
G R I=\frac{G_{1}}{T_{1}}+\frac{G_{2}}{T_{2}}+\frac{G_{3}}{T_{3}} \ldots \frac{G_{n}}{T_{n}}
$$

Índice de la tasa de germinación $\left(\mathbf{R}_{\mathbf{5 0}}\right)$ : Expresa la velocidad de germinación en términos del número de días requeridos para que germine el $50 \%$ de las semillas sembradas (7).

Índice de la tasa de germinación $\left(\mathbf{R}_{50}{ }^{\prime}\right)$ : Expresa la velocidad de germinación en términos del día en el cual ha germinado el $50 \%$ del total de semillas que germinó al final del período de observación (7).

Valor pico (PV): Expresa la velocidad de germinación como el máximo cociente derivado de la división del porcentaje de germinación en el número de días (7).

Índice de germinación media diaria (MDG): Expresa la germinación total en términos del número de semillas germinadas durante el tiempo total de la prueba. Este índice puede ser utilizado independientemente del tiempo que dure la prueba, es decir, sin haber alcanzado la germinación total del lote de semillas evaluadas (7).

Valor de la germinación (GV): Combina la germinación media diaria con la velocidad de germinación (7). Se obtuvo a partir de la fórmula:

Tabla 1. Características físico-químicas de los sustratos evaluados.

\begin{tabular}{|c|c|c|c|c|c|c|c|c|c|c|}
\hline \multirow[b]{2}{*}{ Sustrato } & \multicolumn{3}{|c|}{ Granulometria } & \multirow[b]{2}{*}{ pH } & \multirow{2}{*}{$\begin{array}{c}\text { Sat Hum } \\
(\%)\end{array}$} & \multirow{2}{*}{$\begin{array}{c}\mathrm{CE} \\
(\mathrm{dS} / \mathrm{m})\end{array}$} & \multirow[b]{2}{*}{$\begin{array}{l}\text { C.O } \\
(\%)\end{array}$} & \multirow{2}{*}{$\begin{array}{c}\text { CIC } \\
\text { meq/100 }\end{array}$} & \multirow{2}{*}{$\begin{array}{c}\text { Calcio } \\
\text { meq/100 }\end{array}$} & \multirow{2}{*}{$\begin{array}{r}\text { Magnesio } \\
\text { meq/100 }\end{array}$} \\
\hline & $\begin{array}{c}\text { Arena } \\
(\%)\end{array}$ & $\begin{array}{c}\text { Limo } \\
(\%)\end{array}$ & $\begin{array}{c}\text { Arcilla } \\
(\%)\end{array}$ & & & & & & & \\
\hline Suelo local & 41,2 & 29,4 & 29,4 & 5,5 & 2,4 & 0,26 & 4,1 & 33,1 & 8,0 & 3,0 \\
\hline Turba & 0 & 0 & 0 & 7,34 & 658 & 0,94 & - & - & 0,558 & 0,132 \\
\hline $\begin{array}{c}\text { Suelo-humus } \\
1: 1 \mathrm{v} / \mathrm{v}\end{array}$ & 66 & 22 & 12 & 5,60 & 98 & 0,25 & 4,90 & 7,70 & 2,12 & 0,7 \\
\hline Sustrato & $\begin{array}{c}\text { Potasio } \\
\text { meq/100 }\end{array}$ & $\begin{array}{c}\text { Sodio } \\
\text { meq/100 }\end{array}$ & $\begin{array}{c}\text { Mn } \\
\text { ppm }\end{array}$ & $\begin{array}{c}\text { Fe } \\
\text { ppm }\end{array}$ & $\begin{array}{c}\mathrm{Zn} \\
\mathbf{p p m}\end{array}$ & $\begin{array}{c}\mathrm{Cu} \\
\mathrm{ppm}\end{array}$ & $\begin{array}{c}\text { B } \\
\text { ppm }\end{array}$ & $\begin{array}{c}\text { Sulfatos } \\
\text { ppm }\end{array}$ & $\begin{array}{c}\mathrm{N}-\mathrm{NO}_{3} \\
\text { ppm }\end{array}$ & $\begin{array}{c}\text { Fósforo } \\
\text { ppm }\end{array}$ \\
\hline Suelo local & 1,2 & 0,08 & 28 & 185 & 7,4 & 1,1 & 0,89 & - & 6,3 & 40,2 \\
\hline Turba & 0,133 & 0,061 & 0,11 & 0,40 & 0,16 & 0,04 & 0,90 & 2,8 & 39 & $23\left(\mathrm{PO}_{4}\right)$ \\
\hline $\begin{array}{c}\text { Suelo-humus } \\
1: 1 \mathrm{v} / \mathrm{v} \\
\end{array}$ & 0,28 & 0,25 & 13 & 175 & 2,5 & 1,3 & 0,04 & 6,8 & 39 & 1,9 \\
\hline
\end{tabular}




$$
G V=M D G \times P V
$$

En la etapa de vivero, se aplicó un diseño de parcelas divididas con distribución completamente al azar, el factor principal fue el tipo de sustrato con dos niveles: suelo local (S1) y la mezcla suelo local con humus de lombriz $(1: 1 \mathrm{v} / \mathrm{v})(\mathrm{S} 2)$; el factor secundario fue el estado de desarrollo de las plantas en el momento del trasplante con tres niveles: dos hojas (ED1), cuatro hojas (ED 2) y seis hojas (ED3). Cada tratamiento tuvo tres repeticiones de 180 plantas.

Para recopilar la información de la respuesta de crecimiento y desarrollo de las plantas, se realizaron muestreos destructivos de frecuencia semanal. El análisis del crecimiento se realizó con base en el registro de las variables de medición directa:

Longitud total (cm): Se registro con cinta métrica, la longitud de cada planta desde el ápice de la raíz hasta el meristemo apical del tallo.

Número de hojas: Se contó el número de hojas por planta.

Área foliar $\left(\mathbf{c m}^{2}\right)$ : Se tomaron las hojas de cada planta y se midió el área foliar en un medidor marca Li-cor modelo 3100 .

Peso seco por órganos y total (g): Se separó la planta en sus respectivos órganos y se colocaron en bolsas de papel kraft con su respectiva etiqueta. Las muestras fueron colocadas en horno de secado por 48 horas a $60^{\circ} \mathrm{C}$, para luego pesarlas en la balanza analítica y registrar el peso seco de raíz, tallo y hojas, el peso seco total es el resultado de la sumatoria del peso seco de los órganos.

Los índices tenidos en cuenta fueron:

Índice de relación de área foliar $\operatorname{RAF}\left(\mathrm{cm}^{2} \mathbf{g}^{-1}\right)$ : Indica la relación entre el área foliar y el peso seco total de la planta. Este índice muestra la utilización de asimilados en la fotosíntesis con base a la relación de área foliar total y el peso seco total de la planta. Se determina a partir de la fórmula:

$$
R A F=\left[\frac{\left(\frac{A F 1}{P S 1}\right)+\left(\frac{A F 2}{P S 2}\right)}{2}\right]
$$

Donde: $P S$ es el peso seco total, $A F$ es el área foliar de la planta (8).

Índice de relación de peso foliar $\operatorname{RPF}\left(\mathrm{g} \mathrm{g}^{-1}\right)$ : Expresa la relación entre el peso seco de las hojas y el peso seco total de la planta. Este índice establece la distribución de asimilados hacia las hojas y es un indicador de la frondosidad de la planta. Se determinó con la fórmula:

$$
R P F=\left[\frac{\left(\frac{P S A F 1}{P S 1}\right)+\left(\frac{P S A F 2}{P S 2}\right)}{2}\right]
$$

Donde: $P S A F$ es el peso seco del área foliar y $P S$ es el peso seco total (8).

Índice de relación de masa radical $\operatorname{RMR}\left(\mathrm{g} \mathrm{g}^{-1}\right)$ : Indica la relación entre el peso seco de la raíz y el peso seco total de la planta. Este índice determina la distribución de asimilados hacia la raíz. Se determinó con la fórmula:

$$
R M R=\left[\frac{\left(\frac{P S R 1}{P S 1}\right)+\left(\frac{P S R 2}{P S 2}\right)}{2}\right]
$$

Donde: $P S R$ es el peso seco de la raíz y $P S$ es el peso seco total (8).

Área Foliar Específica AFE $\left(\mathbf{c m}^{2} \mathbf{g}^{-1}\right)$ : Expresa la relación entre el área foliar y el peso seco de las hojas. Mide el grosor de la hoja y representa la superficie foliar por gramo de hoja. Se calcula mediante la fórmula:

$$
A F E=\left[\frac{\left(\frac{A F 1}{P S A F 1}\right)+\left(\frac{A F 2}{P S A F 2}\right)}{2}\right]
$$

Donde: $A F$ es el área foliar y $P S A F$ es el peso seco de las hojas (8).

\section{Análisis de Resultados}

Para el procesamiento estadístico de la información obtenida en las etapas de semillero y vivero se realizaron análisis de varianza (ANOVA) con el propósito de determinar las diferencias significativas entre los diferentes tratamientos; a partir de estos resultados se realizó una prueba de intervalos múltiples de Duncan para comparar las semejanzas o diferencias entre las medias de los tratamientos de acuerdo a cada una de las variables e índices evaluados $(\alpha<0,05)$. La información fue procesada con el programa Statistical Analysis System (SAS), utilizando el procedimiento de modelos lineales (GLM). 


\section{Resultados y discusión}

\section{Etapa de Semillero}

Las curvas de germinación en general exhibieron dos etapas definidas: una logarítmica y otra lineal (Figura 1). La etapa logarítmica, correspondió al período entre la siembra y el inicio de la germinación, caracterizada por la progresiva absorción de agua por parte de los distintos tejidos que forman la semilla.

En esta etapa, la tasa de crecimiento de las estructuras del embrión fue lenta y aumentó a medida que transcurrió el tiempo por efecto de la actividad respiratoria $(9,10,11)$; presentó una duración de ocho días para las semillas sembradas en los tratamientos S1P1 (Suelo local y siembra directa al azar), S1P2 (suelo local y sembrado a $0,5 \mathrm{~cm}$ de profundidad), S2P1 (Suelo local con humus y siembra directa al azar) y S2P2 (suelo local con humus y sembrado a $0,5 \mathrm{~cm}$ de profundidad); y una duración de dos días para S3P1 (turba y siembra directa al azar) y S3P2 (turba y sembrado a $0,5 \mathrm{~cm}$ de profundidad).
En la etapa lineal de la germinación de las semillas, la radícula emergió a medida que transcurrió el tiempo, destacándose que el porcentaje de germinación fue diferente en las semillas de los tratamientos: en S1P1, S1P2, S2P1 y S2P2 fue cercano al 50\% y en S3P1 y S3P2 fue de 76 y $84,3 \%$ respectivamente (Figura 1). En la etapa lineal de la germinación ocurren transformaciones metabólicas requeridas para el desarrollo del embrión, la tasa de crecimiento del embrión se incrementa a una velocidad constante, y es el periodo en el que germina la mayor parte de las semillas $(9,12)$.

El ANOVA realizado determinó diferencias altamente significativas por efecto de los sustratos para todos los índices de germinación (Tabla 2).

\section{Efecto del Sustrato}

Los resultados de la prueba de Duncan (Tabla 3) muestran que los tres sustratos evaluados influenciaron diferencias estadísticas definiendo tres grupos: grupo A para la

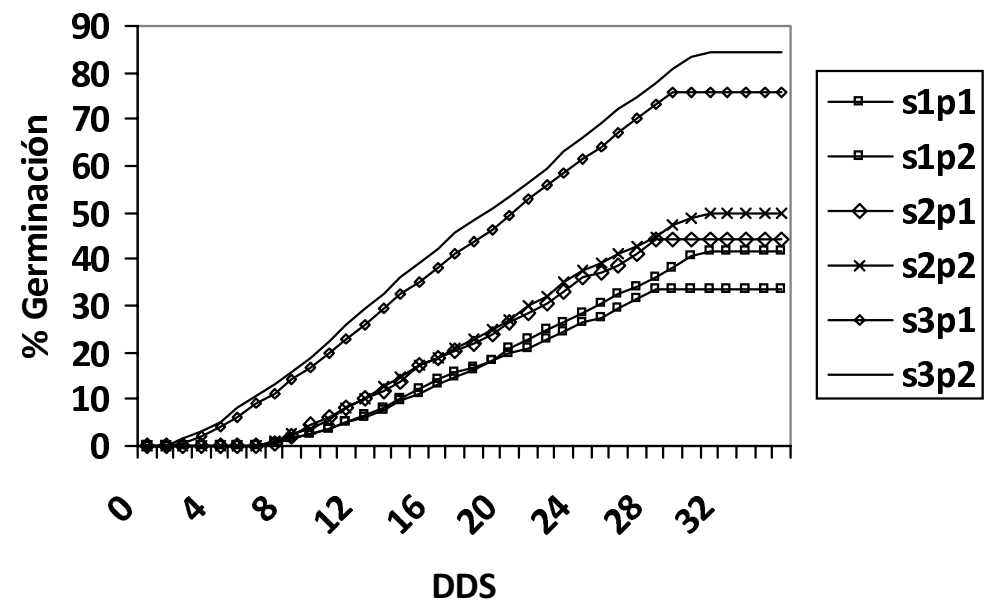

Figura 1. Porcentaje de germinación de las semillas de M. quadrangularis, sembradas en diferentes sustratos y arreglos espaciales.

Tabla 2. Análisis de varianza para los índices de germinación de acuerdo al tipo de sustrato y arreglo espacial utilizado en el semillero de $M$. quadrangularis .

\begin{tabular}{lllllllll}
\hline F. V & GL & GC & $\mathbf{R}_{\mathbf{5 0}}$ & $\mathbf{R}_{\mathbf{5 0}}$ & GRI & PV & MGD & GV \\
\hline Sustrato & 2 & $30,12 * *$ & $25,69 * *$ & $21,50^{*}$ & $532,93 * *$ & $3,63 * *$ & $21,26 * *$ & $17,81^{* *}$ \\
Arreglo espacial & 1 & $0,22 \mathrm{~ns}$ & $1,36 \mathrm{~ns}$ & $1,38 \mathrm{~ns}$ & $578,00 \mathrm{~ns}$ & $0,013 \mathrm{~ns}$ & $0,020 \mathrm{~ns}$ & $0,98 \mathrm{~ns}$ \\
Sustrato*arreglo & 2 & $30,89 \mathrm{~ns}$ & $1,36 \mathrm{~ns}$ & $0,056 \mathrm{~ns}$ & $358,166 \mathrm{~ns}$ & $0,051 \mathrm{~ns}$ & $2,14 \mathrm{~ns}$ & $2,30 \mathrm{~ns}$ \\
Promedio & & 70,98 & 26,53 & 23,36 & 21,68 & 2,40 & 1,65 & 4,10 \\
CV (\%) & & 6,44 & 6,74 & 8,65 & 14,68 & 7,21 & 6,44 & 29,36 \\
\hline
\end{tabular}

$\mathrm{ns}=$ no significativo, $*=$ Significancia $(\mathrm{P}<0,05), * *=$ Significancia $(\mathrm{P}<0,01)$. 
Tabla 3. Efecto de los tres tipos de sustrato sobre los índices de germinación en semillas de M. quadrangularis.

\begin{tabular}{lllllllr}
\hline Sustrato & \multicolumn{7}{c}{ Índices de germinación } \\
\hline & GC & \multicolumn{1}{c}{$\mathbf{R}_{\mathbf{5 0}}$} & $\mathbf{R}_{\mathbf{5 0}} \mathbf{0}^{-}$ & GRI & PV & MDG & GV \\
\hline Suelo & $40,83 \mathrm{c}$ & 0 & $18,83 \mathrm{a}$ & $24,16 \mathrm{c}$ & $1,36 \mathrm{c}$ & $1,36 \mathrm{c}$ & $1,87 \mathrm{c}$ \\
Suelo local*humus & $50,83 \mathrm{~b}$ & $30 \mathrm{a}$ & $18,83 \mathrm{a}$ & $50,83 \mathrm{~b}$ & $1,71 \mathrm{~b}$ & $1,69 \mathrm{~b}$ & $2,91 \mathrm{~b}$ \\
Turba & $83,66 \mathrm{a}$ & $18,5 \mathrm{~b}$ & $15,33 \mathrm{~b}$ & $83,66 \mathrm{a}$ & $2,85 \mathrm{a}$ & $2,78 \mathrm{a}$ & $7,98 \mathrm{a}$ \\
\hline
\end{tabular}

Promedios con letras distintas indican diferencia significativa según la prueba de Duncan.

1

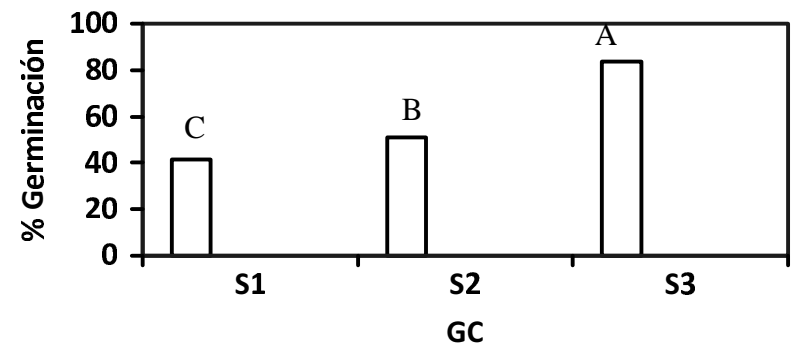

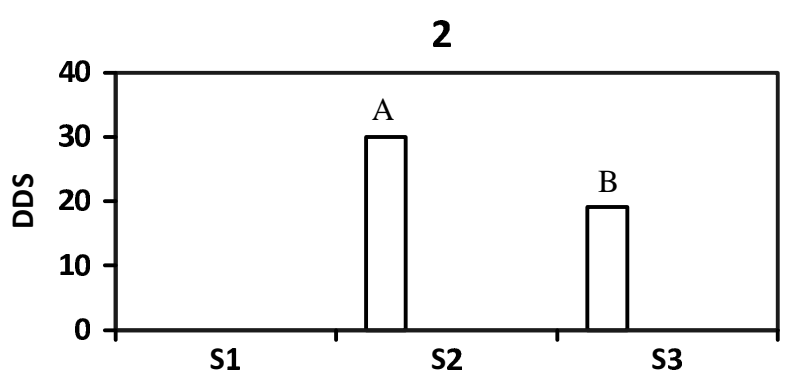

3

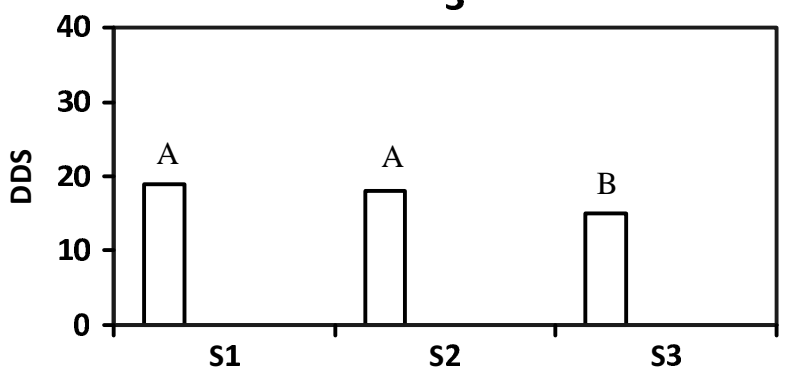

Figura 2. Índices de germinación 1. GC, 2. R y 3. R 'evaluados en semillas de $M$. quadrangularis en diferentes tipos de sustratos. Los promedios con letras distintas ${ }^{50}$ indican ${ }^{50}$ diferencia significativa según la prueba de Duncan $(P=0,05)$.

turba (S3), sustrato en el cual las semillas presentaron la mayor capacidad germinativa (GC) con $83,66 \%$, y los mayores valores en los índices GRI, PV, MDG y GV; el grupo B, para la suelo local con humus (S2) con un GC de $50,83 \%$ y el grupo $C$, para el suelo local (S1) en el cual se obtuvo la menor capacidad germinativa con un $40,83 \%$ y los menores valores para los índices de germinación en el sustrato suelo (Figura 2).

Para el índice $\mathrm{R}_{50}$, se definieron dos grupos estadísticos: el grupo A, para la turba $(\mathrm{S} 3)$, se requirieron de menos días, 18.5 DDT, para obtener el 50\% de semillas germinadas, mientras para el grupo B, la mezcla suelo local con humus (S2), se obtuvo el 50\% de germinación a los 30 DDT; el suelo local no se incluyó en algún grupo debido a que no se obtuvo el $50 \%$ de la germinación de las semillas sembradas. Para el índice $\mathrm{R}_{50}$, la prueba de Duncan, definió dos grupos: el grupo A, incluye los sustratos suelo local (S1) y suelo local con humus (S2), en estos se necesitó de 18 días para obtener el $50 \%$ de las semillas germinadas respecto al sustrato turba, grupo $\mathrm{B}$, que requirió 15 días (Figura 2, 3). En la supervivencia y germinación de las semillas de arboloco, la textura del sustrato es importante, debido a que asegura condiciones de humedad y aireación a la semilla; los suelos muy compactos reducen el suministro de agua y oxigeno (13), necesarios para los procesos metabólicos como la respiración celular, la actividad enzimática, la síntesis y reparación de DNA, RNA y la 
síntesis de proteínas requeridos para inducir el crecimiento y desarrollo del embrión $(14,15,16,17)$.

\section{Efecto del arreglo espacial}

Los índices de germinación GC, PV, MDG y GV de las semillas esparcidas por siembra directa al azar (P1) y a 0.5 $\mathrm{cm}$ de profundidad (P2) no presentaron diferencias estadísticas, la germinación de semillas de Arboloco, no fue afectada por la profundidad de siembra (Figura 3), lo que se puede explicar teniendo en cuenta, que en los arreglos espaciales se presentaron las mismas variaciones en el flujo del agua; la semilla del arboloco es muy pequeña y queda expuesta a las condiciones del medio $(16,18)$. Bragachini et al. (2002) afirman que las semillas deben colocarse a una profundidad que no sobrepase el doble del tamaño de la semilla; para que ésta reciba el oxígeno necesario para germinar. Cuando las semillas son sembradas a profundidades excesivas, mueren y en el caso que logren germinar, agotan todas las reservas antes de emerger, sin embargo, sí se siembran demasiado superficiales existe el riesgo de que la semilla se deshidrate y muera.

Los índices $\mathrm{GC}, \mathrm{R}_{50}, \mathrm{R}_{50}{ }^{\prime}$, GRI, PV, MDG y GV presentaron los mayores valores en cuanto velocidad, uniformidad, tasa y capacidad germinativa en los arreglos espaciales en los que se utilizó turba (S3P1 y S3P2), (7).

\section{Efecto de la interacción entre el sustrato y el arreglo espacial}

Las semillas de los tratamientos (S3P1 y S3P2) presentaron las mejores respuestas germinativas en los índices $\mathrm{GC}, \mathrm{R}_{50}$, $\mathrm{R}_{50}{ }^{\prime}$, GRI, PV, MDG y GV debido a que presentaron los mayores valores en cuanto a velocidad, uniformidad, tasa y capacidad germinativa (Figura 3), (7); estos resultados pueden deberse a la alta capacidad de retención de agua de la turba, lo que permitió la rápida rehidratación de las semillas y por consiguiente la reanudación de la actividad respiratoria y la activación de enzimas que participan en el metabolismo respiratorio y en los procesos de síntesis de ácidos nucléicos y proteínas $(10,20)$.

\section{Etapa de Vivero}

La prueba de ANAVA determinó diferencias estadísticas para las variables de respuesta: altura de la planta, longitud de la raíz, área foliar, peso seco de por órganos y total y en los índices de crecimiento: RAF, AFE, RMR y RPF, por efecto del sustrato, estado de desarrollo y la interacción estado de desarrollo por sustrato (Tabla 4).

\section{Efecto del sustrato}

Los resultados del ANAVA (Tabla 4), indican que los sustratos y los estados de desarrollo influenciaron

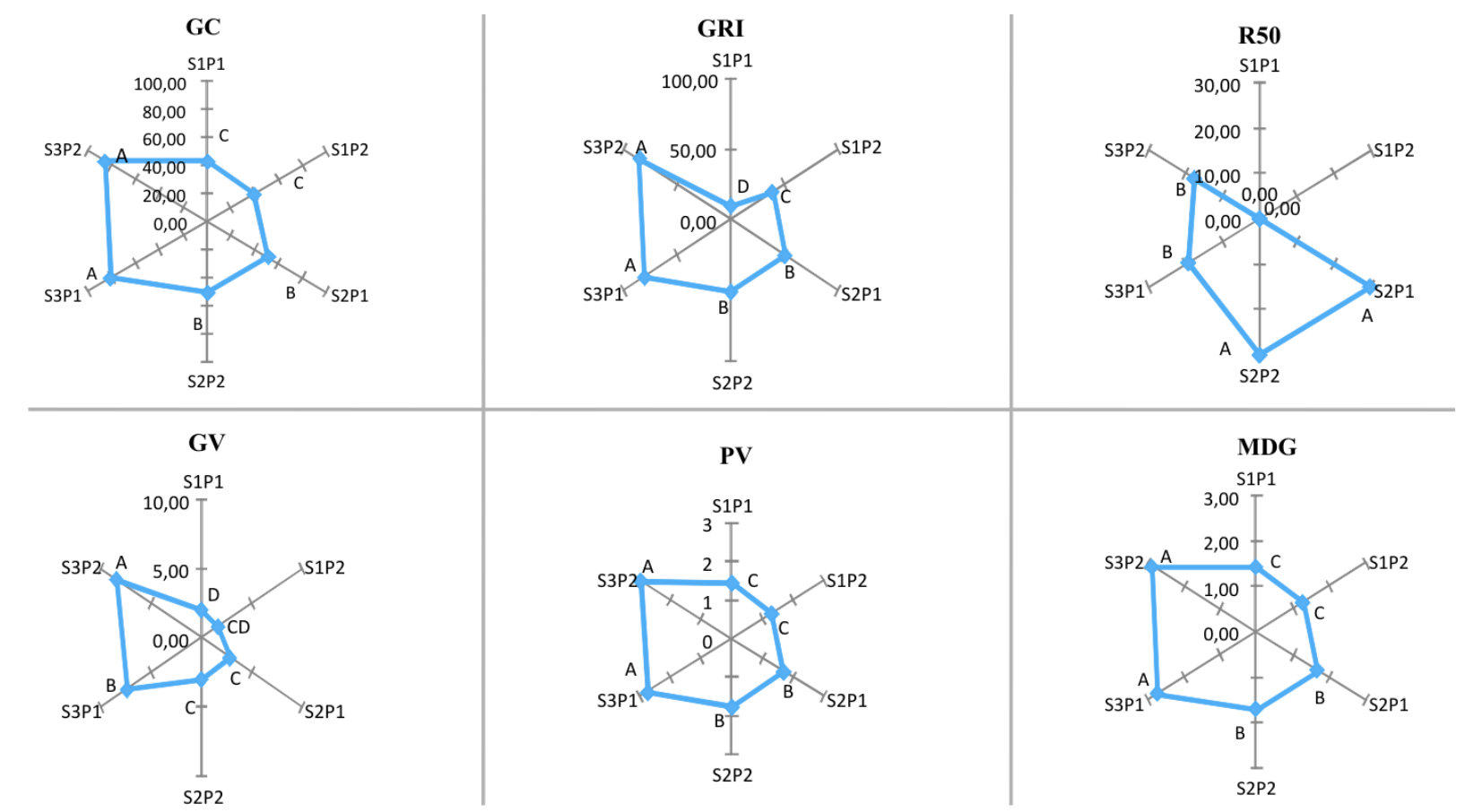

Figura 3. Índices de germinación GC, GRI, R, GV, PV y MGD de semillas de M. quadrangularis en los sustratos asociados a arreglos espaciales. 
diferencias estadísticas en la respuesta de las plantas de arboloco. La prueba de Duncan definió dos grupos para los sustratos evaluados: el grupo A, para la mezcla suelo local con humus de lombriz (S2) y el grupo B para el suelo local (S1) en las variables de respuesta: longitud total de la planta, longitud de la raíz, número de hojas, área foliar, peso seco del tallo, hojas y peso seco total, siendo el mejor sustrato, la mezcla tierra local con humus (S2), (Tabla 5).

Los valores más altos para todas las variables de respuesta e índices de crecimiento se obtuvieron el suelo local con humus de lombriz (S2), mientras en las plantas sembradas en suelo local, se encontraron los valores más bajos (Tabla 5); este resultado se puede explicar al considerar que el humus de lombriz es un sustrato que aporta carbono orgánico y elementos minerales esenciales en cantidades requeridas para responder las necesidades de crecimiento de las plantas de arboloco; así mismo interviene en la fertilidad física del suelo porque aumenta la superficie activa y también, mejora la aireación y aumenta la capacidad de retención de agua y nutrientes del suelo (30). Las respuestas de las plantas que crecen bajo condiciones limitantes, como la poca disponibilidad de nutrientes en el suelo, se relacionan con la reducción de los procesos de organogénesis y morfogénesis de la planta, debido a que los minerales participan en el metabolismo vegetal, cumpliendo funciones especificas como constituyentes de estructuras orgánicas, activadores enzimáticos de de reacciones, portadores de carga y osmoreguladores (21, $22,23,24)$.

\section{Efecto de los estados de desarrollo}

Según los resultados del ANAVA (Tabla 4), los estados de desarrollo determinaron diferencias significativas entre los promedios de las variables de respuesta y los índices de crecimiento evaluados. La prueba de Duncan (Tabla 5), definió en general tres grupos: el grupo A para las plantas con seis hojas (ED3), el B para las plantas con cuatro hojas (ED2) y el C para las plantas con dos hojas (ED1). En las variables: número de hojas, área foliar, peso secos por órganos y total, se definieron dos grupos: el grupo A para las plantas que se trasplantaron con seis hojas y el grupo B para las plantas trasplantadas con dos y con cuatro hojas.

De igual manera se definieron tres grupos para los índices de crecimiento RAF, AFE y RPF: el grupo A para ED1, el B para ED2 y el C para ED3. Los resultados obtenidos indican que las plantas trasplantadas con dos hojas (ED1) presentan suficiente tejido foliar, característica favorable para las plantas en el momento del trasplante, debido a que la superficie foliar con sólo dos hojas, asegura la interceptación y aprovechamiento de la radiación fotosintéticamente activa, lo que favorece la fotosíntesis y producción de foto asimilados requeridos en los procesos de crecimiento y desarrollo de las plantas $(25,26,27,21)$. En las plantas, los órganos que actúan como fuentes de fotoasimilados son las hojas, estas tienen la capacidad para fotosintetizar y producir los carbohidratos que los órganos demanda de la planta utilizarán para soportar el crecimiento. En este sentido, las plantas de arboloco con dos hojas permitieron suplir las necesidades de los órganos demanda, en estos se aprovecharon los fotoasimilados producidos para el crecimiento y el desarrollo (26).

\section{Efecto de la interacción entre el sustrato y el estado de desarrollo}

Los resultados de las variables de crecimiento de las plantas de arboloco variaron según el efecto del sustrato y de los estados de desarrollo, incrementándose en función del tiempo. Las variables de respuesta longitud total y área foliar (Figura 4A y 4B) durante la etapa vegetativa inicial, presentaron tendencias de tipo sigmoide, típicas del crecimiento vegetal, que se caracterizan por presentar tres etapas: la etapa logarítmica, la etapa lineal y la etapa de

Tabla 4. ANAVA para las variables de respuesta y los índices de crecimiento en los sustratos y estados de desarrollo $M$. quadrangularis.

\begin{tabular}{|c|c|c|c|c|c|c|c|c|c|c|c|c|c|}
\hline F.V. & $\begin{array}{l}\mathbf{G} \\
\mathbf{L}\end{array}$ & $\begin{array}{l}\text { Long } \\
\text { total }\end{array}$ & $\begin{array}{l}\text { Long } \\
\text { raíz }\end{array}$ & $\begin{array}{l}\mathrm{N}^{\circ} \\
\text { hojas }\end{array}$ & $\begin{array}{l}\text { Área } \\
\text { foliar }\end{array}$ & PST & PSR & PSA & PSP & RAF & AFE & RPF & RMR \\
\hline E D & 2 & $94,4 * *$ & $15,9^{* *}$ & $20,1 * *$ & $70,9 * *$ & $0,004 *$ & $0,005^{* *}$ & $0,009 * *$ & $0,05^{* *}$ & $14,1 * *$ & $87,6^{* *}$ & $0,09 * *$ & $0,011 \mathrm{~ns}$ \\
\hline $\mathrm{S}$ & 1 & $38,8 * *$ & $56,14 * *$ & $12,7 * *$ & $58,6^{* *}$ & $0,03^{* *}$ & $0,0023^{*}$ & $0,05^{* *}$ & $0,21 * *$ & $24,9 \mathrm{~ns}$ & $32,4^{*}$ & $0,124^{*}$ & $0,05^{*}$ \\
\hline $\mathrm{ED}^{*} \mathrm{~S}$ & 2 & $13,3^{*}$ & $11,56^{* *}$ & $18,3^{* *}$ & $24,8 \mathrm{~ns}$ & $0,003^{*}$ & $0,001 \mathrm{~ns}$ & $0,003^{*}$ & $0,017^{*}$ & $50,6^{* *}$ & 10,3 & $0,078^{*}$ & $0,11 * *$ \\
\hline C.V. & & 14,96 & 21,65 & 13,23 & 28,6 & 11,1 & 11,5 & 28,8 & 20,8 & 27,7 & 16,6 & 21,6 & 32,64 \\
\hline
\end{tabular}

$\mathrm{ED}=$ Estado de desarrollo, $\mathrm{S}=$ sustrato, $\mathrm{ns}=$ no significativo, $*=$ Significancia $(\mathrm{P}<0,05), * *=$ Significancia $(\mathrm{P}<0.01)$. 
Tabla 5. Efecto del sustrato y estado de desarrollo sobre el crecimiento de M. quadrangularis.

\begin{tabular}{|c|c|c|c|c|c|c|c|c|c|c|c|c|}
\hline Sustrato & $\begin{array}{l}\text { Longitud } \\
\text { total }\end{array}$ & $\begin{array}{l}\text { Long } \\
\text { Raíz }\end{array}$ & $\begin{array}{c}\mathbf{N}^{\circ} \\
\text { Hojas }\end{array}$ & $\begin{array}{l}\text { Área } \\
\text { Foliar }\end{array}$ & $\begin{array}{c}\text { P Seco } \\
\text { tallo }\end{array}$ & $\begin{array}{c}\text { P Seco } \\
\text { Raíz }\end{array}$ & $\begin{array}{l}\text { P Seco } \\
\text { Hojas }\end{array}$ & $\begin{array}{c}\text { P Seco } \\
\text { Total }\end{array}$ & RAF & AFE & RPF & RMR \\
\hline S1 & $7,89 \mathrm{~b}$ & $11,62 b$ & $5,49 \mathrm{~b}$ & $16,51 b$ & $0,01 \mathrm{~b}$ & $0,01 \mathrm{a}$ & $0,03 b$ & $0,06 \mathrm{~b}$ & $280,4 \mathrm{a}$ & $691,5 \mathrm{a}$ & $0,42 \mathrm{a}$ & $0,25 \mathrm{a}$ \\
\hline S2 & $9,51 \mathrm{a}$ & $13,59 \mathrm{a}$ & $6,43 a$ & $36,69 \mathrm{a}$ & $0,03 \mathrm{a}$ & $0,02 \mathrm{a}$ & $0,04 \mathrm{a}$ & $0,10 \mathrm{a}$ & $293,5 \mathrm{a}$ & $706,5 \mathrm{a}$ & $0,45 \mathrm{a}$ & $0,23 a$ \\
\hline $\begin{array}{l}\text { Estado } \\
\text { desarrollo }\end{array}$ & $\begin{array}{c}\text { Longitud } \\
\text { total }\end{array}$ & $\begin{array}{l}\text { Long } \\
\text { Raíz }\end{array}$ & $\begin{array}{c}\mathbf{N}^{\circ} \\
\text { Hojas }\end{array}$ & $\begin{array}{l}\text { Área } \\
\text { Foliar }\end{array}$ & $\begin{array}{c}\text { P Seco } \\
\text { tallo }\end{array}$ & $\begin{array}{c}\text { P Seco } \\
\text { Raíz }\end{array}$ & $\begin{array}{l}\text { P Seco } \\
\text { Hojas }\end{array}$ & $\begin{array}{l}\text { P Seco } \\
\text { Total }\end{array}$ & RAF & AFE & RPF & RMR \\
\hline D1 & $7,94 \mathrm{c}$ & $11,82 \mathrm{c}$ & $5,74 \mathrm{~b}$ & $22,13 b$ & $0,02 b$ & $0,01 b$ & $0,03 b$ & $0,07 \mathrm{~b}$ & $377,7 \mathrm{a}$ & $925,5 \mathrm{a}$ & $0,46 \mathrm{a}$ & $0,24 a$ \\
\hline D2 & $8,84 \mathrm{~b}$ & $12,40 \mathrm{~b}$ & $5,81 \mathrm{~b}$ & $24,05 b$ & $0,02 b$ & $0,014 \mathrm{~b}$ & $0,03 b$ & $0,07 \mathrm{~b}$ & $277,63 \mathrm{~b}$ & $674,05 \mathrm{~b}$ & $0,44 \mathrm{~b}$ & $0,25 \mathrm{a}$ \\
\hline D3 & $9,31 \mathrm{a}$ & $13,59 a$ & $6,33 a$ & $33,67 \mathrm{a}$ & $0,03 a$ & $0,02 \mathrm{a}$ & $0,04 \mathrm{a}$ & $0,10 \mathrm{~b}$ & $206,12 \mathrm{c}$ & $498,9 c$ & $0,41 \mathrm{c}$ & $0,23 a$ \\
\hline
\end{tabular}

Promedios con letras distintas indican diferencia significativa según la prueba de Duncan $(P=0,05)$.
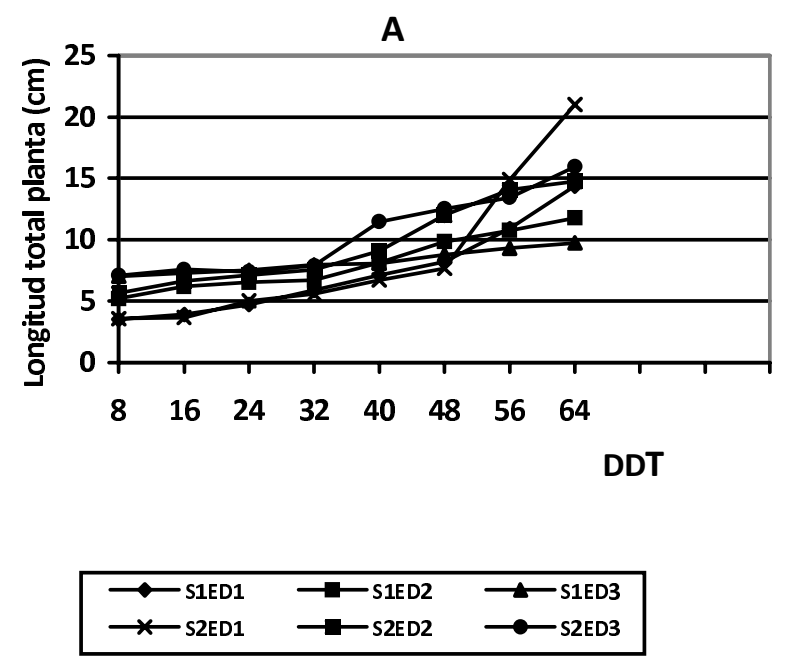

B
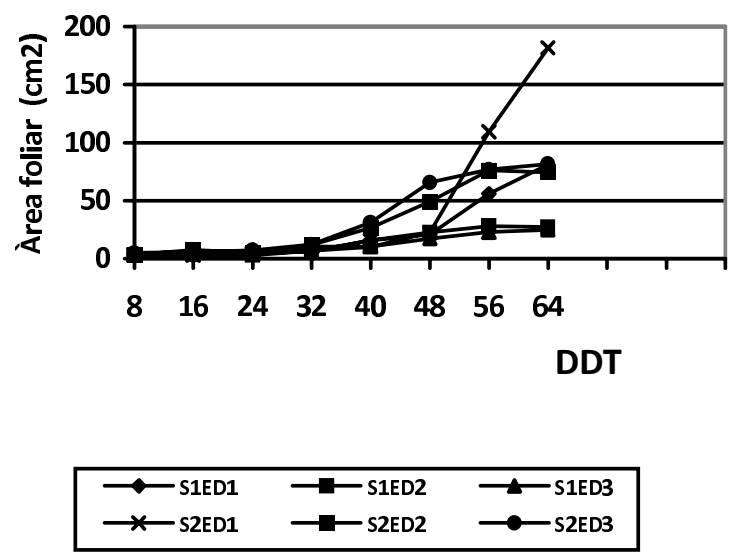

Figura 4. Comportamiento de las variables A. Longitud total por planta y B. Área foliar de las plantas de M. quadrangularis en diferentes sustratos.

senescencia. La etapa logarítmica ocurrió entre los 8 y los 32 DDT, en esta el tamaño de los órganos y estructuras vegetales aumentó en forma exponencial con el tiempo, por lo cual la tasa de crecimiento fue baja al comienzo, pero aumentó de manera constante. La etapa lineal ocurrió desde los 32 hasta los 64 DDT, incrementándose el tamaño de los órganos a una velocidad constante en el tiempo $(21,27,28)$.

En plantas perennes, como árboles es probable que se presenten curvas sigmoidales repetidas en el tiempo ya que estos por lo general son de crecimiento indeterminado (12).
Los resultados más altos para las variables de respuesta longitud total por planta y área foliar a los 64 DDT (Figuras 4A y 4B respectivamente), se obtuvieron en el sustrato suelo local con humus y en plantas trasplantadas con dos hojas (S2ED1) con $21 \mathrm{~cm}$, mientras en el suelo local y plantas con dos hojas (S1ED1) se presentó el valor más bajo de longitud con $9,71 \mathrm{~cm}$. Con respecto al área foliar, en plantas del tratamiento S2ED1 se observó el valor más alto a los 64 DDT con $181,44 \mathrm{~cm}^{2}$ en comparación con S1ED3 que tuvo el valor más bajo de $24,95 \mathrm{~cm}^{2}$; este resultado se puede explicar teniendo en cuenta que la absorción de nutrientes aportados por el lombricompuesto representó un efecto positivo en el crecimiento del tallo 
primario y de las hojas de las plantas en las primeras etapas de desarrollo, en las que se demanda una mayor cantidad de nutrientes minerales que soporten el crecimiento vegetal $(29,30)$, (Figura 4).

Los valores del índice de Relación Área Foliar aumentaron a través del tiempo en las plantas de arboloco de todos los tratamientos, observándose que al transplantar con dos hojas en lombricompuesto (S2ED1) se presentó el valor más alto a los 64 DDT con $515,55 \mathrm{~cm}^{2} \mathrm{~g}^{-1}$ respecto a las plantas del tratamiento en el que se utilizó suelo local y plantas de seis hojas (S1ED3) que presentó el valor más bajo con 145,35 $\mathrm{cm}^{2} \mathrm{~g}^{-1}$ (Figura 5A). El RAF es una medida del balance entre la capacidad fotosintética potencial y el costo respiratorio potencial y evalúa la relación entre el área foliar total y el peso seco total, permitiendo describir la utilización de las materias fotosintetizadas dentro de la planta $(8,31,32)$.

El índice AFE hace referencia al área foliar promedio de una hoja abierta por unidad de peso foliar y mide la densidad de hojas o el grosor relativo de una capa de hojas (8). Los valores más altos del AFE a los 64 DDT se encon-

A

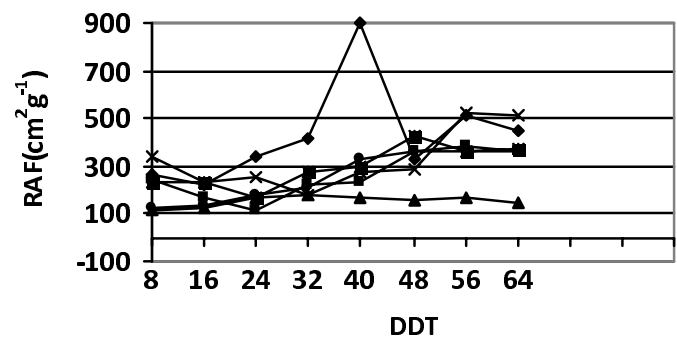

C

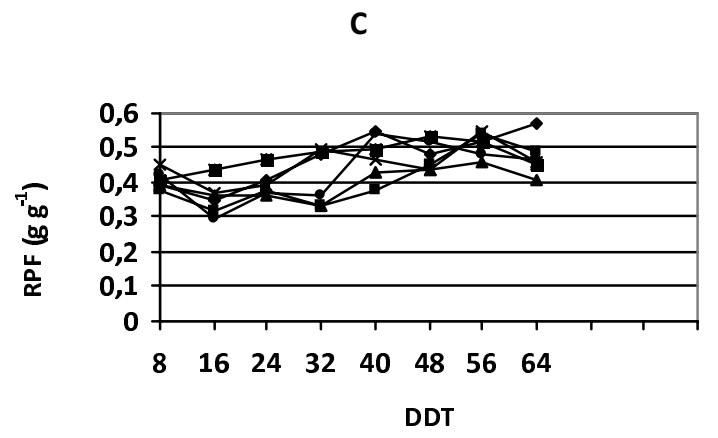

traron en las plantas de los tratamientos en los que se utilizó suelo local mezclado con humus y plantas con cuatro hojas (S2ED2) con 1126,12 $\mathrm{cm}^{2} \mathrm{~g}^{-1}$, mientras en las plantas del tratamiento suelo local mezclado con humus y plantas con seis hojas (S1ED3) se presentó el valor promedio más bajos de $362,41 \mathrm{~cm}^{2} \mathrm{~g}^{-1}$. El AFE expresa el grosor relativo de la capa de hojas de la planta y se incrementa a medida que hay un mayor sombreamiento. Cuando el valor de AFE es muy alto, se afirma que la tasa respiratoria de la planta está aumentando, pero si se presenta un valor bajo es la tasa fotosintética la que se encuentra alta y por consiguiente la acumulación de materia seca en hojas también (23).

Para la Relación de Peso Foliar (RPF) se observa que los valores más altos se obtuvieron en la última semana de muestreo 64DDT en general en las plantas de todos los tratamientos evaluados, en las plantas trasplantadas en suelo local con humus de lombriz y plantas con dos hojas (S1ED1) se obtuvo el valor más alto $0,57 \mathrm{~g} \mathrm{~g}^{-1}$ (Figura 5C). El RPF es un índice de la producción de hojas de la planta con base en el peso seco total y se relaciona con la distribución de materia seca de este órgano a lo largo del
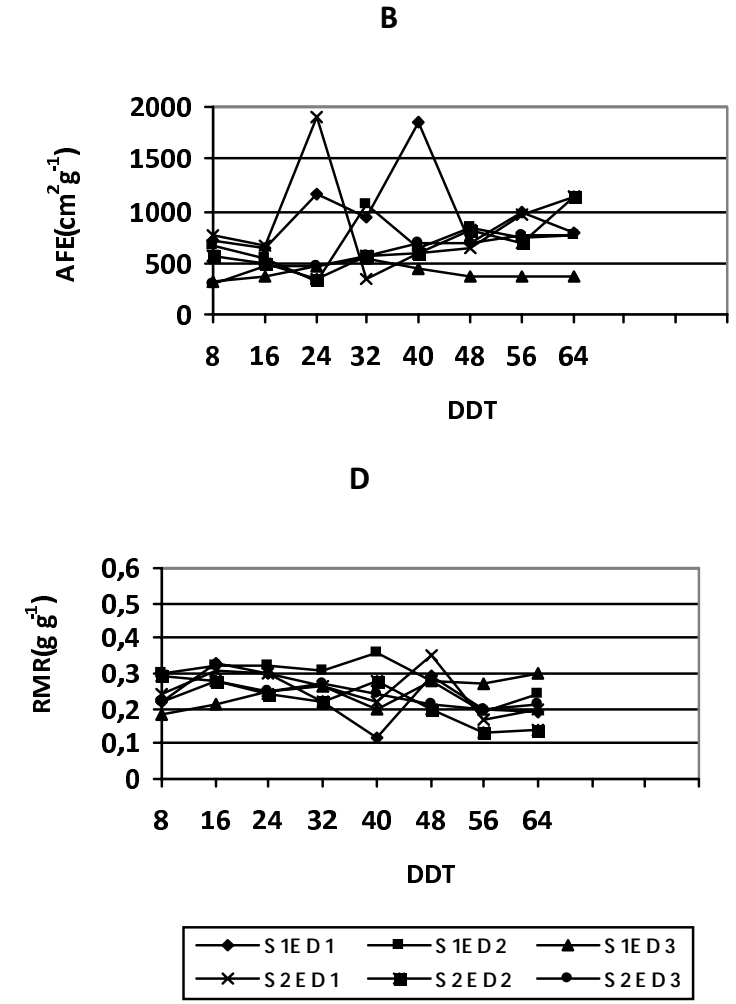

Figura 5. Índices de crecimiento A. RAF, B. AFE, C.RPF y D. RMR en plantas de M. quadrangularis respecto a los sustratos y estados de desarrollo. 
ciclo del cultivo, indicando que aumenta la distribución de biomasa para la formación de nuevas hojas. Se observa que los valores más altos de RPF se obtienen en las últimas semanas de muestreo debido a que la planta se encuentra en el estadio principal de crecimiento de hojas y destina los asimilados a la formación de dichos órganos. Se espera que este índice siga aumentando en el tiempo hasta que la fuerza de la demanda se dirija a la formación de los órganos de la etapa reproductiva de la planta $(23,24,33)$.

El índice que refleja la distribución de materia seca hacia la raíz sobre el peso total de la planta (RMR), indicó que a los 64 DDT, las plantas sembradas en el sustrato suelo local y plantas con cuatro hojas (S1ED2) y en suelo local y plantas con seis hojas (S1ED3) obtuvieron los valores más altos $\left(0,24 \mathrm{~g} \mathrm{~g}^{-1}\right.$ y $\left.0,3 \mathrm{~g} \mathrm{~g}^{-1}\right)$; este resultado puede deberse a que las plantas de arboloco, destinaron gran cantidad de fotoasimilados para la formación de raíces, como un mecanismo de resistencia contra el estrés hídrico causado. El tipo de suelo en el que fueron sembradas las plantas, presentó un alto porcentaje de partículas de arena (66\%) (Tabla 1), condición que evito la compactación del suelo y favoreció el engrosamiento de la raíz $(16,23,33,34)$.

\section{Conclusiones}

El tipo de sustrato utilizado en semillero para la germinación de las semillas de $M$. quadrangularis afecta la capacidad germinativa de estas; el mejor comportamiento se observó en el semillero en el que se utilizó turba. Los arreglos espaciales, en este caso, las profundidades en las que se sembraron no presentaron diferencias pero se recomienda la siembra directa de semillas al azar ya que no demanda tanto tiempo.

El tipo de sustrato utilizado en vivero afecta de manera significativa las respuestas de crecimiento y desarrollo de la planta de $M$. quadrangularis; la mezcla de suelo local con humus de lombriz 1:1 v/v aporta condiciones que favorecen los procesos fisiológicos, así mismo es determinante para obtener una mejor productividad.

El estado de desarrollo en el cual se trasplantaron las plantas afecto sustancialmente el crecimiento y desarrollo de las plantas de arboloco en vivero. En este trabajo se obtuvo que el estado de desarrollo que presentaba mayor número de hojas, seis hojas, presentó los mejores resultados al momento de evaluar el crecimiento y desarrollo de las plantas.

\section{Financiación}

Esta investigación fue financiada parcialmente por el Centro de Investigaciones y Estudios en Biodiversidad y Recursos Genéticos (CIEBREG). El trabajo hace parte del proyecto «Valoración de los bienes y servicios de la biodiversidad para el desarrollo sostenible de paisajes rurales colombianos, Complejo Ecorregional de los Andes del Norte (CEAN)».

\section{Conflicto de intereses}

Los autores declaran que no existen conflictos de intereses relacionados con este trabajo.

\section{Referencias}

1. Fersain A. Maravillas vegetales: El Arboloco o pauche. Centro Internacional de Agricultura Orgánica 1999; 5 (36):1-7.

2. Álvarez L. Biología, uso y manejo del arboloco (Montanoa quadrangularis). Primera edición. Centro editorial Universidad de Caldas. Manizales, Colombia. 2003, 316p.

3. Ministerio del Medio Ambiente. Logros y Avances de la Gestión Ambiental en Colombia: Informe de Gestión- Bogotá, Colombia. 2002, 346p.

4. Gustavo T C. Respuesta de la germinación de semillas de Esenbeckia leiocarpa Engl. (guarantã) al osmocondicionamient. Revista Forestal 2004; 1 (3):1-13.

5. International Seed Testing Association (ISTA). International rules for seed testing. ISTA. Zurich, Switzerland. 2006, 333 p.

6. Thomson A, Kassaby A. Interpretation of seeds germiantion paremeters. New Forest 1993; 7:123-132.

7. Czabator FJ. Germination: An index combining speed and completeness of pine. Seed germination. Forest Science 1962; 8: 386-396 pp.

8. Hunt R, Causton DR, Shipley B, Askew P. A Modern Tool for Classical Plant Growth Analysis. Annals of Botany 2002; 90: 485-488.

9. Barcelo-Coll J, Sabater B, Sánchez R, Rodrigo G. Fisiología Vegetal. Ediciones Pirámide. Madrid, España. $1983,813 p p$. 
10. Baskin CC, Baskin M. Seeds, ecology, biogeography, and evolution of dormancy and germination. First Edition. Academic Press. California, Estados Unidos. 1998, 666 p.

11. Bewley JD. Seed germination and plant dormancy. Plant Cell 1997; 9: 1055-1066.

12. Pérez F, Martínez JB. Introducción a la Fisiología Vegetal. Ediciones Mundi-Prensa. Barcelona, España. 1994, 213 p.

13. Morales C. La Diversidad como Activo para el Desarrollo. "Existe Suficiente Oferta de Abonos Orgánicos para la Agricultura en el Perú”. Pucalpa, Perú. 2003, 12 p.

14. Verdonick O, De Vleeschauwer D, De Boudt M. The Influence of the Substrate to plant grouwth. Acta Horticulture 1981; 126: 251-258.

15. Willumsen J. Improvement of the physical conditions in peat substrates during the germination of cabbage seeds in organic farming. Acta Horticulture 1997; 450: 183-190.

16. Burés S. Sustratos. Agrotécnicas. Madrid, España. 1997, $342 \mathrm{p}$

17. Cantliffe DJ. Seed Germination for Trasplants. Hortechnology 1998; 8 (4): 499-503.

18. Besnier F. Semillas: biología y tecnología. Primera edición. Ediciones mundi-prensa. Madrid, España. 1989, 637 p.

19. Bragachini M, Alfaro M, Mendez A, Pacioni F, VonMartini A. Siembra de maíz, eficiencia de implantación y su efecto sobre la producción de grano. Tercer taller internacional de agricultura de precisión del cono sur de América. Córdoba, Argentina. 2002, 10pp.

20. Copeland AD, McDonald MB. (Ed.). Principles of seed science and technology. Third Edition. Kluwer Academic Publishers Boston. Boston, London.1995, 369 p.

21. Taiz L, Zeiger E. Plant Physiology. Cuarta Edición. Sunderland Massachusetts. 2006, 792pp.

22. Luttge U. Botánica. MacGraw-Hill Interamericana. Madrid, España. 1997, 573p.

23. Lambers H, Chapin F y Pons T. Plant physiological ecology. Springer-verlag. New York, Estados Unidos. 1998, 540 p.
24. Gil A, Orozco J, Gutiérrez E, Espinoza A, Rodríguez S. Análisis de crecimiento de variedades de algodón transgénicas y convencionales. Revista Agraria 2004; 1 (3): 125-130.

25. Muñoz J D, Martínez L J, Giraldo R. Variabilidad espacial de propiedades edáficas y su relación con el rendimiento en un cultivo de papa (Solanum tuberosum L.). Agronomía Colombiana 2006; 24 (2):355-366.

26. Cabezas M. Asignación y distribución de la materia seca en plantas cultivadas, con énfasis en especies leñosas. Universidad de Ciencias Aplicadas y Ambientales: Actualidad \& Divulgación Científica 2006; 9 (1): $3-20$.

27. Larcher W. Physical Plant Ecology. Springer ed . Berlin, Alemania. 2002. 252pp.

28. Azcon-Bieto J, Talón JM. Fundamentos de Fisiología Vegetal McGraw-Hill Interamericana. Madrid, España. 2008, 522 pp.

29. Simpson K. Abonos y Estiércoles. Ed. Acribia S.A. Zaragoza, España. 1986, 265p.

30. Quevedo A. Influencia del humus de lombricultura en el crecimiento inicial de cedro colorado en plantación a campo abierto y comportamiento al ataque de Hypshiphylla sp. Folia Amazónica 1993; 5 (1): 47-57.

31. Gómez C. Ecofisiología de papa (Solanum tuberosum) utilizada para el cultivo fresco y para la industria. Revista Comalfi, 1999; XXVI. (1-3): 82-92.

32. Valbuena R. Evaluación de las Metodologías para el Cálculo de los índices de Crecimiento en dos Variedades de Remolacha (Beta vulgaris) Early Wonder y Detroit. Tesis de Maestría (Fitotecnia). Facultad de Agronomía. Universidad Nacional de Colombia, Bogotá, 1995, 115pp.

33. Paz V, Vera A, Páez A. Distribución de biomasa de Barleria lupulina Lindl. en respuesta a tres regímenes de riego. Facultad de Agronomía 2003; 20 (3): 1-9.

34. Schmidt L. 2000. Seed testing (Chapter 11). In: Guide to handling of tropical and subtropical forest seed. http://www.dfsc.dk. Consultado el 18 de noviembre de 2009. 